\title{
Hábitos alimentarios de niños en edad escolar y el papel de la educación para la salud
}

\author{
Eating habits in school-age children and the \\ health education paper
}

\begin{abstract}
The role of the family, media advertising and school influence on the eating habits of children was reviewed. The exposure of children to these behavioral factors has modified the consumption patterns in children, resulting in an increase in the prevalence of obesity. This underlines the importance of health education as a preventive and promotional tool in the change of behaviors related to the adoption of healthy eating habits, emphasizing the development of nutritional programs applied in schools with the cooperation of parents and teachers to modify unhealthy behaviors and to acquire healthy life styles.

Key words: feeding; childhood; nutritional programs; television publicity; infantile obesity.
\end{abstract}

\section{INTRODUCCIÓN}

La presente revisión de hábitos alimentarios en niños y el papel que juega la educación para la salud en la adquisición de estos hábitos, se deriva ante la problemática que existe entre la modificación de estos y el aumento de la obesidad en niños; así como la asociación de la obesidad como factor de riesgo de padecer enfermedades crónico degenerativas (diabetes, enfermedades cardiovasculares e hipertensión arterial) durante la edad adulta.

El objetivo de esta revisión permite establecer a la educación para la salud como una herramienta preventiva y promocional que a través de la implementación de programas contribuye a la adopción de hábitos alimentarios saludables en esta edad.

Considerando que la alimentación y la nutrición son procesos influenciados por aspectos biológicos, ambientales y socioculturales y que durante la infancia contribuyen a un desarrollo y crecimiento óptimo, así como una maduración biopsicosocial, es necesario que los niños adquieran durante esta etapa hábitos alimentarios saludables. Sin embargo, para ello es necesario considerar factores de tipo fisiológicos, sociales y familiares, donde estos últimos ejercen una fuerte influencia en los patrones de consumo. No obstante, los hábitos alimentarios se han ido modificando por factores que alteran la dinámica familiar tales como la menor dedicación y falta de tiempo para cocinar y la pérdida de autoridad en cuanto a la cantidad y calidad de los alimentos que consumen los niños.
Adriana Ivette Macias $M$. Lucero Guadalupe Gordillo S. Esteban Jaime Camacho $R$.

Unidad Académica Profesional Nezahualcóyotl, Universidad Autónoma del Estado de México, México.

Dirigir la correspondencia a: Profesora

Adriana Ivette Macias Martínez Unidad Académica Profesional Nezahualcóyotl Universidad Autónoma del Estado de México. Av. Bordo de Xochiaca s/n. Colonia Benito Juárez Nezahualcóyotl, Estado de México. México. Fono: 51126426 E-mail: adrivet_msmz@hotmail.com

Este trabajo fue recibido el 25 de Marzo de 2011, aceptado con modificaciones el 20 de Enero de 2012 y aceptado para ser publicado el 10 de Julio de 2012.

La publicidad televisa también ha contribuido a la modificación de los hábitos alimentarios de los niños debido a que representan un grupo de edad acrítico y fácil de manipular en cuanto al consumo de nuevos alimentos, los que la mayor parte de las veces no son saludables.

Considerando la problemática presente la escuela juega un rol fundamental en la promoción de factores protectores en materia de hábitos alimentarios por lo que resulta un campo de acción en la implementación de programas nutricionales. Sin embargo, para que estos programas logren modificar las conductas alimentarias no saludables se requiere de una herramienta capaz de lograr dicho objetivo y la más indicada es la educación para la salud, en donde la colaboración entre los profesionales de este campo, los profesores, los padres de familia y las autoridades sanitarias constituye una estrategia para promover hábitos alimentarios saludables y por lo tanto estilos de vida saludables que perduren hasta la etapa adulta, disminuyendo el riesgo de desarrollar enfermedades crónicodegenerativas.

\section{ALIMENTACIÓN Y NUTRICIÓN EN LA EDAD ESCOLAR}

El estudio de la alimentación y la nutrición ha sido abordado desde las ciencias exactas enfocadas a aspectos fisiológicos y las ciencias sociales que incluyen los hábitos y costumbres alimentarias de cada sociedad (1). En ambos procesos intervienen factores biológicos, socioculturales, psicológicos y ambientales $(2,3)$. En el caso de la alimentación 
como un proceso social, esto no sólo permite al organismo adquirir sustancias energéticas y estructurales necesarias para la vida (4), sino también son símbolos que sirven para analizar la conducta (5).

Por otro lado, la nutrición como proceso aporta energía para el funcionamiento corporal, regula procesos metabólicos y ayuda a prevenir enfermedades $(6,7)$. Desde 2005 la llamada tridimensionalidad de la nutrición establece las relaciones entre los alimentos y los sistemas biológicos, sociales y ambientales de cada sociedad (8).

Los procesos antes mencionados cumplen dos objetivos primordiales durante la infancia: 1) conseguir un estado nutritivo óptimo, mantener un ritmo de crecimiento adecuado y tener una progresiva madurez biopsicosocial y 2) establecer recomendaciones dietéticas que permitan prevenir enfermedades de origen nutricional que se manifiestan en la edad adulta pero que inician durante la infancia (9). Es en esta etapa cuando se adquieren los hábitos alimentarios que tendrán durante toda su vida; sin embargo, también es una etapa de gran variabilidad $(10,11)$ debido a la presencia de factores como: el desarrollo económico, avances tecnológicos, la incorporación de la mujer al ámbito laboral, la gran influencia de la publicidad y la televisión, la incorporación más temprana de los niños a la escuela y la mayor posibilidad por parte de los niños de elegir alimentos con elevado aporte calórico y baja calidad nutricional (12).

Los padres tienen una gran influencia sobre los hábitos alimentarios de los niños y son ellos los que deben decidir la cantidad y calidad de los alimentos proporcionados durante esta etapa; en conjunto con los padres, la escuela (principalmente profesores) juega un papel importante en el fomento y adquisición de hábitos alimentarios saludables a través de la promoción y educación para la salud (9).

\section{HÁBITOS ALIMENTARIOS}

Definir a los hábitos alimentarios no resulta sencillo ya que existe una diversidad de conceptos, sin embargo, la mayoría converge en que se tratan de manifestaciones recurrentes de comportamiento individuales y colectivas respecto al qué, cuándo, dónde, cómo, con qué, para qué se come y quién consumen los alimentos, y que se adoptan de manera directa e indirectamente como parte de prácticas socioculturales $(13,14)$.

En la adopción de los hábitos alimentarios intervienen principalmente tres agentes; la familia, los medios de comunicación y la escuela (15). En el caso de la familia, es el primer contacto con los hábitos alimentarios ya que sus integrantes ejercen una fuerte influencia en la dieta de los niños y en sus conductas relacionadas con la alimentación (16), y cuyos hábitos son el resultado de una construcción social y cultural acordada implícitamente por sus integrantes (17).

Los hábitos alimentarios se aprenden en el seno familiar y se incorporan como costumbres (18), basados en la teoría del aprendizaje social (19) e imitadas de las conductas observadas por personas adultas que respetan (20). Otros modos de aprendizaje se dan a través de las preferencias o rechazos alimentarios en los niños, en donde estos últimos son expuestos repetidamente a una serie de alimentos que conocen a través del acto de comer enmarcado por encuentros entre padres e hijos (21).

Sin embargo, los hábitos alimentarios se han ido modificando por diferentes factores que alteran la dinámica e interacción familiar; uno de ellos corresponde a la situación económica que afecta los patrones de consumo tanto de los niños como de los adultos (22), la menor dedicación y falta de tiempo para cocinar, lo que provoca que las familias adopten nuevas formas de cocina y de organización (23) y la pérdida de autoridad de los padres en la actualidad, ha ocasionado que muchos niños coman cuándo, cómo y lo qué quieran (24).

Por su parte, la publicidad televisiva forma parte del ambiente social humano, que en el caso de su influencia en los hábitos alimentarios de los niños ha ido desplazando a instancias como la familia y la escuela; promoviendo un consumo alimentario no saludable, ya que los niños son más susceptibles de influenciar, debido a que se encuentran en una etapa de construcción de su identidad (25), y por lo tanto son fácilmente manipulables por los anuncios publicitarios que promocionan nuevos alimentos (26).

En el caso de la escuela, dicha institución permite al niño enfrentarse a nuevos hábitos alimentarios que en muchas ocasiones no son saludables; aunque también asume un rol fundamental en la promoción de factores protectores en cuestión de hábitos alimentarios. En este sentido, las acciones de promoción y prevención escolar están a cargo de los profesores a través de los contenidos temáticos en materias como ciencias naturales. Sin embargo, es necesario tratar este tipo de temas desde una perspectiva integral (27) que permita combinar conocimientos, actitudes y conductas saludables que promueva en los niños un estilo de vida saludable (28), e incluso coadyuve a evitar la aparición de síntomas de trastornos alimentarios (29).

\section{LA EDUCACIÓN PARA LA SALUD Y HÁBITOS ALIMENTARIOS}

La educación para la salud como disciplina surge ante la necesidad de proporcionar a las personas los medios necesarios para mejorar la salud, a través de la modificación de conductas de riesgo. En la $36^{a}$ Asamblea Mundial de la Salud se definió a la educación para la salud como cualquier combinación de actividades de información y educación que lleve a una situación en la que las personas sepan cómo alcanzar niveles de salud óptimos y busquen ayuda cuando lo necesiten (30). La educación para la salud tiene como objetivo primordial diseñar programas de intervención destinados a modificar creencias, costumbres y hábitos no saludables, además de promover, proteger y fomentar la salud (31).

Por lo anterior, la educación para la salud como estrategia en las escuelas contribuye a fomentar hábitos alimentarios saludables que contrarresten la prevalencia de obesidad a través de la promoción de hábitos alimentarios y estilos de vida saludables en los escolares (32), que inculque en los niños actitudes, conocimientos y hábitos saludables que favorezcan su crecimiento y desarrollo, el fomento de su salud y la prevención de las enfermedades evitables de su edad (33).

Con base a lo anterior, en México se han realizado algunos esfuerzos por implementar programas nutricionales, basados en la promoción y educación para la salud, uno de ellos el Programa de Acción Específico 2007 - 2012 Escuela y Salud (34) consiste en un programa aplicado por parte de los profesores a través de los temas curriculares en las instituciones escolares de nivel básico y responde a una estrategia integral que identifica varios determinantes de salud, siendo algunos de ellos la alimentación correcta y la actividad física. En este sentido su objetivo reside en impulsar conocimientos en materia de alimentación para generar hábitos y cambios en los niños para disminuir el incremento de la obesidad.

Sin embargo, la implementación de este programa no ha mostrado los resultados esperados (35), por lo que en 2010 
se implementa el Acuerdo Nacional para la Salud Alimentaria (ANSA) que es una estrategia contra el sobrepeso y obesidad infantil y cuya función es establecer los lineamientos para el expendio y distribución de alimentos y bebidas en dichos establecimientos. Por otra parte, en el marco de esta estrategia se deriva el Programa de Acción en el Contexto Escolar; que promueve una cultura de salud mediante el desarrollo de competencias para una vida saludable a través de la participación de diversos sectores, entre los que destaca los profesores, padres de familia, alumnos y encargados de los expendios de alimentos dentro de las escuelas; a través de la promoción y educación para la salud, el fomento de la actividad física regular y el acceso y disponibilidad de alimentos y bebidas que faciliten una alimentación correcta (36).

Con base a lo anterior; las escuelas de nivel básico en México han adoptado la estrategia de "activación física", que consiste en realizar 30 minutos de ejercicio moderado dentro de la institución; por su parte, algunas empresas de la industria alimentaria han reducido las porciones de algunos alimentos que son vendidos en las llamadas "cooperativas escolares", aunque en este sentido la mayoría de dichos alimentos son frituras, jugos embotellados o pastelitos y en cuanto al fomento de hábitos alimentarios, dicha función se encuentra a cargo de los profesores.

Como se puede observar, se han hecho algunos esfuerzos por disminuir la prevalencia de obesidad en los niños mexicanos; sin embargo, no todas las instituciones de educación básica las han adoptado. Por lo que es necesario que los profesionales en el campo de la educación para la salud se incorporen a la implementación de este tipo de programas; o al diseño de programas que contribuyan a fomentar hábitos alimentarios saludables y por ende crear estilos de vida saludables.

\section{RESUMEN}

Se realizó una revisión teórica acerca del papel de la familia, los anuncios publicitarios y la escuela, en la adopción, o bien modificación de los hábitos alimentarios de los niños. Debido a que las nuevas dinámicas familiares y la gran exposición de los niños a los comerciales alimentarios han modificado los patrones de consumo de los niños originando con ello el aumento de la obesidad infantil, motivo por el que se enfatiza la importancia de la educación para la salud como herramienta preventiva y promocional en el cambio de conductas relacionadas con la adopción de hábitos alimentarios saludables, destacando el desarrollo de programas nutricionales aplicados en escuelas con el objetivo de buscar el trabajo conjunto entre los padres de familia y los profesores, para lograr modificar conductas no saludables y lograr estilos de vida saludables.

Palabras clave: alimentación; infancia; programas nutricionales; publicidad televisa; obesidad infantil.

\section{BIBLIOGRAFÍA}

1. Gracia M. La complejidad biosocial de la alimentación humana. Zainak 2000; 20: 35-55.

2. Contreras J, Gracia M. Alimentación y cultura. Perspectivas antropológicas. Ariel, España, 2005.

3. Macias A. I, Quintero M. L, Camacho E. J, Sánchez J. M. La tridimensionalidad del concepto de nutrición: su relación con la educación para la salud. Rev Chil Nutr 2009; 36: 1129-35.

4. Ortiz A. S, Vázquez V, Montes M. La alimentación en México: enfoques y visión a futuro. Estud Soc 2005; 13: 8-25.
5. Carrasco S. Orientaciones teóricas y formulación de problemas en el estudio socioantropológico de la alimentación. Alteridades 2003; 13: 105-13.

6. Gómez C, De Cos A. I. Nutrición en atención primaria. Novartis, España, 2001.

7. Pinto A, Carbajal A. La dieta equilibrada, prudente o saludable. Instituto de Salud Pública, Madrid, 2003.

8. Beauman C, Cannon G, Elmadfa I, Glasauer P, Hoffmann I, Keller $M$, et al. The principles, definition and dimensions of the new nutrition science. Public Health Nutr 2005; 8: $695-8$

9. Lozano M. C. Condicionantes socioeconómicos de los hábitos alimentarios e ingesta de energía y nutrientes en escolares de la población española. Universidad Complutense de Madrid, España, 2003.

10. Tojo $R$, Leis R. Alimentación del niño escolar. Manual práctico de nutrición en pediatría. Ergon, Madrid, 2007.

11. Macedo $E$, Valverde $M$, López $R$, Rodríguez $A$, López $C$, Delgado $C$, et al. Manual. Cómo mejorar en la escuela la alimentación de niñas, niños y las y los adolescentes. Dirección General de Promoción de la Salud, México, 2007.

12. Hidalgo M. I. Nutrición en la edad preescolar, escolar y adolescente. Pediatría Integral 2003; 7: 340-54.

13. Moreiras O, Cuadrado C. Hábitos alimentarios. Tojo, Barcelona, 2001.

14. Ortiz A. S, Vázquez V, Montes M. La alimentación en México: enfoques y visión a futuro. Estud Soc 2005; 13: 8-25.

15. Serra L. L, Ribas L, Pérez C, García C, Peña L, Aranceta J. Determinants of nutrient intake among children and adolescents: Results from the EnKid Study. Ann Nutri and Metab 2002; 46: 31-38.

16. Moreno J. M, Galiano M. J. La comida en familia: algo más que comer juntos. Acta Pediátr Española 2006; 64: 554-8.

17. García M, Pardío J, Arroyo P, Fernández V. Dinámica familiar y su relación con hábitos alimentarios. Estud Cult Contemp 2008; 14: 9-46.

18. Guerrero N, Campos O. I, Luengo J. Estudios sobre hábitos alimentarios racionales de los niños y jóvenes, con especial incidencia en la población inmigrante. Confederación de Consumidores y Usuarios (CECU), España, 2005.

19. Bandura, A. Social foundations of thought and action: A social cognitive theory. Prentice-Hall, Estados Unidos, 1986.

20. López-Alvarenga J. C, Vázquez-Velázquez V, Bolado-García J, Castañeda-López J, Robles L, Velásquez-Alva C, et al. Influencia de los padres sobre las preferencias alimentarias en niños de dos escuelas primarias con diferente estrato económico. Estudio ESFUERSO. Gac Méd Méx 2007; 143: 463-9.

21. Busdiecker S, Castillo C, Salas I. Cambios en los hábitos de alimentación durante la infancia: una visión antropológica. Rev Chil Pediatr 2000; 71: 37-46.

22. Restrepo S. L, Maya M. La familia y su papel en la formación de los hábitos alimentarios en el escolar. Un acercamiento a la cotidianidad. Bol de Antropol 2005; 19: 127-48.

23. Sandoval S. A, Domínguez S, Cabrera A. De golosos y tragones están Ilenos los panteones: cultura y riesgo alimentario en Sonora. Estud Soc 2010; 17: 150-79.

24. Bolaños P. Evolución de los hábitos alimentarios. De la salud a la enfermedad por medio de la alimentación. Trast Condu Alime 2009; 9: 956-72.

25. Sauri M. C. Publicidad televisiva, hábitos alimentarios y salud en adolescentes de la ciudad de Mérida, Yucatán, México. Centro de Investigación y de Estudios Avanzados 
del Instituto Politécnico Nacional Unidad Mérida, México, 2003.

26. Herrero C. El horario infantil en la televisión: de la falta de imaginación a la irresponsabilidad de los mensajes publicitarios. Trast Cond Aliment 2008; 7: 752-66.

27. De Vincezi A, Tudesco F. La educación como proceso de mejoramiento de la calidad de vida de los individuos y de la comunidad. Rev Iberoamer Educ 2007; 49: 1-12.

28. Fernández V. Escuela y medios de comunicación: ¿Lógicas diferenciales en la transmisión de la cultura común y la construcción de la ciudadanía?. Rev Iberoamer Educ 2007; 4: 1-10.

29. Escoto M. C, Mancilla J. M, Camacho E. J. A pilot study of the clinical and statistical significance of a program to reduce eating disorder risk factors in children. Eat Weig Disor, 2008; 13: 111-18.
30. Perea Q. R, Bouche P. H. Educación para la salud: reto de nuestro tiempo. Díaz Santos, España, 2004.

31. Valadez I, Villaseñor M, Alfaro N. Educación para la Salud: la importancia del concepto. Rev Educ Des 2004; 33: 43-8.

32. Bonzi N. S, Bravo M. Patrones de alimentación en escolares: Calidad versus cantidad. Rev Méd Rosario 2008; 74: 48-57.

33. Robles M. Prensa y educación para la salud en la escuela. Comunicar 1996; 6: 94-9.

34. Cuevas L, Mundo V, Shamah T, Ávila M. A. (2010). Programas de mejoramiento dentro de las escuelas. Instituto Nacional de Salud Pública, México, 2010.

35. Secretaría de Salud. Programa de Acción Específico 2007 - 2012. Escuela y Salud. Subsecretaría de Prevención y Promoción de la Salud, México, 2006.

36. Ponce E. Programa de Acción en el Contexto Escolar. Secretaría de Educación Pública, México, 2010. 